\title{
Article \\ Dysregulation of Synaptic Signaling Genes Is Involved in Biology of Uterine Leiomyoma
}

\author{
Jovan Krsteski ${ }^{1}$, Mario Gorenjak ${ }^{1}\left(\mathbb{D}\right.$, Igor But ${ }^{2}$ (D) Maja Pakiž ${ }^{2}$ and Uroš Potočnik ${ }^{1,3, *(\mathbb{D}}$ \\ 1 Centre for Human Molecular Genetics and Pharmacogenomics, Faculty of Medicine, University of Maribor, \\ Taborska ulica 8, 2000 Maribor, Slovenia; jkrstevski@yahoo.com (J.K.); mario.gorenjak@um.si (M.G.) \\ 2 Department of General Gynecology and Gynecological Urology, University Clinical Centre Maribor, \\ Ljubljanska ulica 5, 2000 Maribor, Slovenia; but.igor@gmail.com (I.B.); majapakiz@gmail.com (M.P.) \\ 3 Laboratory of Biochemistry, Molecular Biology and Genomics, Faculty of Chemistry and Chemical \\ Engineering, University of Maribor, Smetanova 17, 2000 Maribor, Slovenia \\ * Correspondence: uros.potocnik@um.si; Tel.: +38-62-234-58-54
}

Citation: Krsteski, J.; Gorenjak, M.; But, I.; Pakiž, M.; Potočnik, U. Dysregulation of Synaptic Signaling Genes Is Involved in Biology of Uterine Leiomyoma. Genes 2021, 12, 1179. https://doi.org/10.3390/ genes12081179

Academic Editor: Albert Jeltsch

Received: 9 June 2021

Accepted: 28 July 2021

Published: 29 July 2021

Publisher's Note: MDPI stays neutral with regard to jurisdictional claims in published maps and institutional affiliations.

Copyright: (c) 2021 by the authors. Licensee MDPI, Basel, Switzerland. This article is an open access article distributed under the terms and conditions of the Creative Commons Attribution (CC BY) license (https:// creativecommons.org/licenses/by/ $4.0 /)$.

\begin{abstract}
Uterine leiomyomas are tumors, which are hormone driven and originate from the smooth muscle layer of the uterine wall. In addition to known genes in leiomyoma pathogenesis, recent approaches also highlight epigenetic malfunctions as an important mechanism of gene dysregulation. RNA sequencing raw data from pair-matched normal myometrium and fibroid tumors from two independent studies were used as discovery and validation sets and reanalyzed. RNA extracted from normal myometrium and fibroid tumors from 58 Slovenian patients was used as independent confirmation of most significant differentially expressed genes. Subsequently, GWA data from leiomyoma patients were used in order to identify genetic variants at epigenetic marks. Gene Ontology analysis of the overlap of two independent RNA-seq analyses showed that NPTX1, NPTX2, CHRM2, $D R D 2$ and $C A C N A 1 A$ were listed as significant for several enriched GO terms. All five genes were subsequently confirmed in the independent Slovenian cohort. Additional integration and functional analysis showed that genetic variants in these five gene regions are listed at a chromatin structure and state, predicting promoters, enhancers, DNase hypersensitivity and altered transcription factor binding sites. We identified a unique subgroup of dysregulated synaptic signaling genes involved in the biology and pathogenesis of leiomyomas, adding to the complexity of tumor biology.
\end{abstract}

Keywords: uterine leiomyomas; NPTX1; NPTX2; CHRM2; DRD2; CACNA1A

\section{Introduction}

Uterine leiomyomas (UL), also known as uterine fibroids, are fibroid tumors that are hormone driven and originate from the smooth muscle layer of the uterine wall [1,2]. It is believed that the growth of UL is promoted by gonadal hormones, especially estrogen [3]. The tumors are characterized by deposition of extracellular matrix, which is disorganized and results in various sizes of benign neoplasms [4,5]. The estimated prevalence of UL ranges from 20 to $77 \%$ [6]. While UL are usually asymptomatic, up to $25 \%$ of women with UL may experience abdominal pain, heavy menstrual bleeding, increased risk of miscarriage or infertility [7]. The only curative treatment is hysterectomy, as non-surgical treatment options do not exist [8]. New genetic techniques have provided new insights into the pathogenesis of UL, with stratification into four main subtypes: mutations of mediator of transcription subunit 12 (MED12), fumarate hydratase (FH), high mobility group AT-hook 2 (HMGA2) translocations and collagen gene deletions [9]. It has been found that approximately $70 \%$ of patients with UL carry mutations in exon 2 of MED12 [10]. Additionally, recent work has also revealed subtype-specific gene expression profiles, which supports the idea of different mechanisms of leiomyoma pathogenesis [11]. Furthermore, growth factors and their cognate receptors also play an important role in the pathogenesis of UL [12]. It was clearly shown that alterations in over-expression of TGF- $\beta$ 
may contribute to the growth of UL $[13,14]$. TGF- $\beta$ stimulation increases extracellular matrix protein production and simultaneously decreases degradation of the matrix in UL [15]. Recent state-of-the-art integrative approaches have identified a wide genetic and epigenetic etiology underlying UL. Integration of transcriptomic and epigenetic changes has allowed for the identification of differential transcription factor occupancy, differential enhancer engagement consisting of histone acetylation, and altered enhancer-promoter contact rewiring as key events in UL gene dysregulation and differential expression [16]. Moreover, the regulatory potential of altered DNA methylation driving UL development has also been shown [17]. Additionally, common loci between UL and other gynecological diseases or phenotypes have also been identified by whole-genome association studies (GWAs) [18,19], implicating a complex background in the etiology of UL and insinuating the interplay of genetic variants and epigenetic expression regulation.

As recent approaches highlight epigenetic malfunctions as important mechanisms of gene dysregulation, we aimed to use our previously published transcriptomic-genomic integration approach $[20,21]$ to identify additional genetic-epigenetic interplay regions involved in the formation and biology of UL. For that, we used the combination and integration of publicly available data from three previously published independent studies and our own cohort. Publicly available RNA sequencing (RNA-seq) raw data reads from pair-matched normal myometrium and fibroid tumors from two independent studies $[16,17]$ were used as discovery and validation sets in the present study. Subsequently, RNA extracted from normal myometrium and fibroid tumors from our own Slovenian cohort was used as independent confirmation of identified genes, and meta-analysis GWA summary [18] was used in order to identify genetic variants at epigenetic marks.

\section{Materials and methods}

\subsection{Subjects}

We enrolled 36 Slovenian patients with clinically diagnosed UL who had undergone surgical treatment at the Department of General Gynecology and Gynecological Urology (University Medical Centre Maribor, Maribor, Slovenia). Out of 36, 14 women presented with solitary and 22 with multiple uterine fibroids. RNA was extracted from solitary tumors and from one tumor of patients with multiple UL. Clinical data are presented in Table 1. Additionally, 22 patients who underwent surgical treatment for non-UL-related conditions (with pelvic organ prolapse) were enrolled as controls in order to extract RNA from normal myometrium. The mean age of enrolled women was $43.3 \pm 6.5$ years for cases and $60.6 \pm 11.5$ years for controls. The study protocol was approved by the National Medical Ethics Committee and the Institutional Review Board (KME 43/10/15). Written informed consent was obtained from all enrolled subjects.

Table 1. Clinical data of enrolled patients with UL.

\begin{tabular}{cc}
\hline Data & Values \\
\hline Age mean (95\% CI) & $43.3(40.6-45.4)$ \\
Positive familial history (\%) & 17.7 \\
Menarche mean (95\% CI) & $12.6(12.1-14.1)$ \\
Pregnancy mean (95\% CI) & $3(2.5-3.5)$ \\
Parity mean (95\% CI) & $1.9(1.6-2.2)$ \\
Miscarriage mean (95\% CI) & $0.6(0.2-1.0)$ \\
Oral contraceptives (\%) & 60 \\
Progestin therapy (\%) & 16 \\
BMI mean (95\% CI) & $26.3(25.0-28.5)$ \\
\hline
\end{tabular}

\subsection{RNA Sequencing Analysis}

RNA-seq analysis was performed using publicly available pair-matched paired-end raw datasets from SRP166862 and SRP217468 [17] and SRP188330 [16] from previously published studies. Datasets SRP166862 and SRP217468 were used as discovery and valida- 
tion sets in the study performed by George and colleagues [17] and were merged to obtain the discovery dataset in the present study. The discovery dataset consisted of 15 matched normal myometrium and fibroid tumors. If multiple tumors were present, samples from other UL were discarded from the analyses. Dataset SRP188330 was used as the validation set in the present study and also consisted of 15 matched normal myometrium and fibroid tumors. Both datasets were analyzed independently using the R 4.0.2 environment (R Core Team 2020, Vienna, Austria). Paired-end reads were mapped to the hg19 reference genome and assigned to genomic features using Rsubread 2.2.4 R package and featureCounts [22,23]. Counts per million (CPMs) were estimated using edgeR 3.30.3 $\mathrm{R}$ package [24] and low expressed genes were filtered out based on CPMs corresponding to read counts of 10 . Retained genes were normalized using the trimmed mean of $\mathrm{M}$ values method [25]. Meanvariance modeling at the observational level transformation (VOOM) was applied [26], and differential expression of fibroid tumors relative to normal myometrium was estimated using models and empirical bayes implemented in limma 3.44.3 R package [27] and using blocking to adjust for paired samples. Differential expression was considered for genes with $q$ value $<0.05$ and loget $>2$ or $<-2\left(\operatorname{loget}=\log _{2}\right.$ (fibroid $/$ normal)).

\subsection{Gene Ontology Analysis}

Gene Ontology analysis was performed using the software package CytoScape 3.8.1 [28] with integrated application ClueGO v2.5.7 [29]. ClueGO analysis was performed using the following parameters and selected options: Ontology/Pathways selected: Biological Process, Cellular Component and Molecular Function Evidence selected: only All_Experimental.

\subsection{Extraction of $R N A$}

RNA was extracted from 25 to $30 \mathrm{mg}$ of fibroid tumors and normal myometrium using a miRNeasy mini kit (QIAGEN, Germantown, ML, USA) after manual homogenization. Purity and concentration of nucleic acids were determined using Synergy 2 spectrophotometer (BioTek, Winooski, VT, USA), and integrity of RNA was checked using agarose gel electrophoresis.

\subsection{Validation Using RT- $q P C R$}

A total of $1 \mu \mathrm{g}$ of mRNA was transcribed into cDNA using a high-capacity cDNA reverse transcription kit (Thermo Fisher, Waltham, MA, USA). Nucleotide sequences of target genes NPTX1, NPTX2, DRD2, CHRM2 and CACNA1A were obtained from the NCBI Nucleotide database (www.ncbi.nlm.nih.gov/nuccore/, accessed on 15 March 2021), and isoform non-specific primers were hand-picked using IDT OligoAnalyzer Tool (eu.idtdna. $\mathrm{com} /$ calc/analyzer, accessed on 15 March 2021). Reference genes primers for ACTB and $B 2 M$ were obtained from a previous study [30]. Primer sequences and accession numbers are summarized in Table 2. Primers were synthesized by Sigma (Merck, Darmstadt, Germany). Reverse transcription quantitative polymerase chain reaction (RT-qPCR) gene expression experiments were carried out using LightCycler 480 SYBR Green I Master Mix and a LightCycler 480 real-time thermocycler (Roche, Basel, Switzerland). An amount of $2 \mu \mathrm{L}$ of 10 -fold diluted cDNA $(5 \mathrm{ng} / \mu \mathrm{L})$ was used as a template. Efficiency was $>90 \%$ for all primer pairs, and specificity of amplification was estimated using melting curves for each sample after each run. Raw $C_{T}$ values were obtained from three run-independent technical replicates for each sample. Geometric averaging of reference genes was used for normalization, and relative expression was calculated using the $2^{-\Delta \Delta \mathrm{Ct}}$ method [31]. Statistical analysis was performed using linear $2^{-\Delta \mathrm{Ct}}$ calculation and binomial generalized linear models adjusted to age in the R environment. 
Table 2. Primer sequences and accession numbers.

\begin{tabular}{cccc}
\hline GENE & ACCESSION & FW $\mathbf{5}^{\prime}$ to $\mathbf{3}^{\prime}$ & ${\text { RV } \mathbf{5}^{\prime} \text { to } \mathbf{3}^{\prime}}^{\prime}$ \\
\hline NPTX1 & NM_002522.4 & GTGGCAGTGGCGAGAACT & GGTCCCAGATGTTGAAGTGG \\
NPTX2 & NM_002523.3 & CAGGACGGAGAGAAGCTG & AGTGGCATCAAACCTACCC \\
CHRM2 & NM_001006630.2 & CTATCAACCCTGCCTGCTAT & ACCTTGTAGCGCCTATGTTC \\
DRD2 & NM_000795.4 & TCCCAGCAGAAGGAGAAGAA & TGTTCAGGATGTGTGTGATGAA \\
CACNA1A & NM_000068.4 & TTGTGGTGTTCCCCTTCTTC & ACATGCGGTACTGGAAGCTC \\
\hline
\end{tabular}

\subsection{Correlation Analyses and Machine Learning Prediction Value Estimation}

In order to perform correlation analyses, raw counts from publicly available RNA-seq data were transformed to transcripts per million (TPM). Both discovery and validation datasets were first merged and divided into normal myometrium and fibroid tumors datasets in order to obtain 30 samples per set. Correlation analyses were performed using the 4.0.2 environment (R Core Team 2020, Vienna, Austria) and using PerformanceAnalytics 2.0.4 R package (github.com/braverock/PerformanceAnalytics). Additionally, all five genes were further assessed using randomForest 4.6-14 [32] $\mathrm{R}$ package and receiver operating characteristics analysis using Proc [33] R package. The assessment was made independently for obtained RNA-seq TPM data and obtained RT-qPCR data.

\subsection{Integration to Meta-GWAs and In Silico Functional Analysis}

Uterine leiomyoma GWA data were obtained from publicly available summary statistics of uterine leiomyoma meta-analysis of cohorts of the Women's Genome Health Study, UK Biobank, Queensland Institute of Medical Research, and the North Finnish Birth Cohort of white European ancestry (www.ebi.ac.uk/gwas/studies/GCST009158, accessed on 5 May 2021) [18]. Summary statistics included summaries for 11,464,556 variants. All variants ranging $\pm 100 \mathrm{~kb}$ from previously identified differentially expressed genes were extracted and further analyzed. Functional analyses were conducted using HaploReg v4.1 [34] and GTEx Portal [35]. Regional Manhattan plots were constructed using LocusZoom [36].

\section{Results}

\subsection{RNA Sequencing and Differential Expression}

Using available pair-matched raw datasets (SRP166862 and SRP217468; merged and used as discovery [17] and SRP188330; used as validation) [16] from previously published studies, we performed our own RNA-seq analysis using the aforementioned pipeline in the $\mathrm{R}$ environment. The discovery dataset was first filtered according to $q$ value $<0.05$ and loget $>2$ or $<-2$, where 294 significantly differentially expressed genes were observed in fibroid tumors relative to normal myometrium (Table S1). Subsequently, the same RNAseq pipeline was applied to the validation cohort, where 443 significantly differentially expressed genes were observed in fibroid tumors relative to normal myometrium, using the same filtering thresholds (Table S1). The results of the validation dataset RNA-seq analysis confirmed 204 significantly differentially expressed genes from the discovery dataset (Figure 1).

\subsection{GO Analysis}

Subsequent Gene Ontology analysis of 204 genes confirmed on the validation dataset showed that 15 significantly enriched terms were listed after Bonferroni correction (Table 3). It was observed that NPTX1 and NPTX2 genes were listed at eight enriched terms, followed by DRD2 and CHRM2, which were listed at seven enriched terms. Interestingly, the $C A C N A 1 A$ gene was listed at four enriched terms where all four aforementioned genes were also present. All five genes were significantly upregulated in both the discovery and validation datasets (Figure 2). Based on that observation, NPTX1, NPTX2, DRD2, CHRM2 and CACNA1A genes were considered for an additional validation using RT-qPCR. 


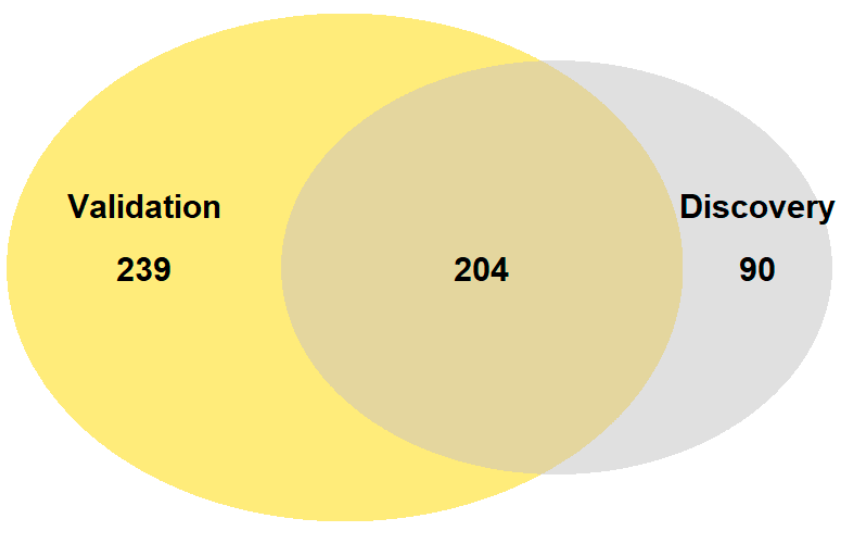

Figure 1. Overlap of significantly expressed genes of discovery and validation RNA-seq analysis.

Table 3. Significant GO terms for 204 confirmed genes.

\begin{tabular}{|c|c|c|c|c|}
\hline GO ID & GO TERM & ONTOLOGY & BONFERRONI $p$ & GENES \\
\hline GO:0098644 & complex of collagen trimers & Cellular component & 0.004 & COL11A1, COL2A1, COL4A4 \\
\hline GO:0009308 & amine metabolic process & Biological process & 0.004 & ATCAY, HDC, INMT, TDO2 \\
\hline GO:0098960 & $\begin{array}{l}\text { postsynaptic neurotransmitter } \\
\text { receptor activity }\end{array}$ & Biological process & 0.006 & $\begin{array}{l}\text { CHRM2, DRD2, } \\
\text { NPTX1, NPTX2 }\end{array}$ \\
\hline GO:0032835 & glomerulus development & Biological process & 0.015 & $B M P 7, C D 24, C O L 4 A 4$ \\
\hline GO:0001822 & kidney development & Biological process & 0.020 & $\begin{array}{l}\text { BMP7, CD24, COL4A4, } \\
\text { OSR1, STRA6 }\end{array}$ \\
\hline GO:0099565 & $\begin{array}{c}\text { chemical synaptic } \\
\text { transmission, postsynaptic }\end{array}$ & Biological process & 0.025 & $\begin{array}{l}\text { CHRM2, DRD2, } \\
\text { NPTX1, NPTX2 }\end{array}$ \\
\hline GO:0072001 & renal system development & Biological process & 0.025 & $\begin{array}{l}\text { BMP7, CD24, COL4A4, } \\
\text { OSR1, STRA6 }\end{array}$ \\
\hline GO:0001655 & $\begin{array}{l}\text { urogenital system } \\
\text { development }\end{array}$ & Biological process & 0.027 & $\begin{array}{c}\text { BMP7, CD24, COL4A4, } \\
\text { OSR1, STRA6 }\end{array}$ \\
\hline GO:0098916 & $\begin{array}{l}\text { anterograde trans- } \\
\text { synaptic signaling }\end{array}$ & Biological process & 0.027 & $\begin{array}{c}\text { CACNA1A, CHRM2, DRD2, } \\
\text { NPTX1, NPTX2 }\end{array}$ \\
\hline GO:0007268 & chemical synaptic transmission & Biological process & 0.027 & $\begin{array}{c}\text { CACNA1A, CHRM2, DRD2, } \\
\text { NPTX1, NPTX2 }\end{array}$ \\
\hline GO:0099537 & trans-synaptic signaling & Biological process & 0.030 & $\begin{array}{c}\text { CACNA1A, CHRM2, DRD2, } \\
\text { NPTX1, NPTX2 }\end{array}$ \\
\hline GO:0030594 & $\begin{array}{l}\text { neurotransmitter } \\
\text { receptor activity }\end{array}$ & Molecular function & 0.031 & $\begin{array}{c}\text { CHRM2, DRD2, GRIA2, } \\
\text { NPTX1, NPTX2 }\end{array}$ \\
\hline GO:0044106 & $\begin{array}{l}\text { cellular amine } \\
\text { metabolic process }\end{array}$ & Biological process & 0.033 & ATCAY, HDC, TDO2 \\
\hline GO:0099536 & synaptic signaling & Biological process & 0.034 & $\begin{array}{c}\text { CACNA1A, CHRM2, DRD2, } \\
\text { NPTX1, NPTX2 }\end{array}$ \\
\hline GO:0010469 & $\begin{array}{l}\text { regulation of signaling } \\
\text { receptor activity }\end{array}$ & Biological process & 0.034 & CRHBP, NPTX1, NPTX2 \\
\hline
\end{tabular}

\subsection{RT-qPCR Validation of GO Identified Genes}

NPTX1, NPTX2, DRD2, CHRM2 and CACNA1A were selected for an additional validation using RT-qPCR. All five genes were upregulated in both discovery and validation datasets (Table 4). Subsequent RT-qPCR analysis was performed using RNA extracted from Slovenian patients, from 22 unmatched normal myometrium and 36 unmatched fibroid tumors. All five genes were proven to be statistically significantly upregulated in fibroid tumors relative to normal myometrium (Table 4; Figure 3). Moreover, the gene expression of NPTX1, NPTX2, CHRM2, DRD2 and CACNA1A was significantly upregulated throughout both RNA-seq and RT-qPCR analyses. Raw RT-qPCR $\mathrm{C}_{\mathrm{T}}$ values are provided in Table S2. 

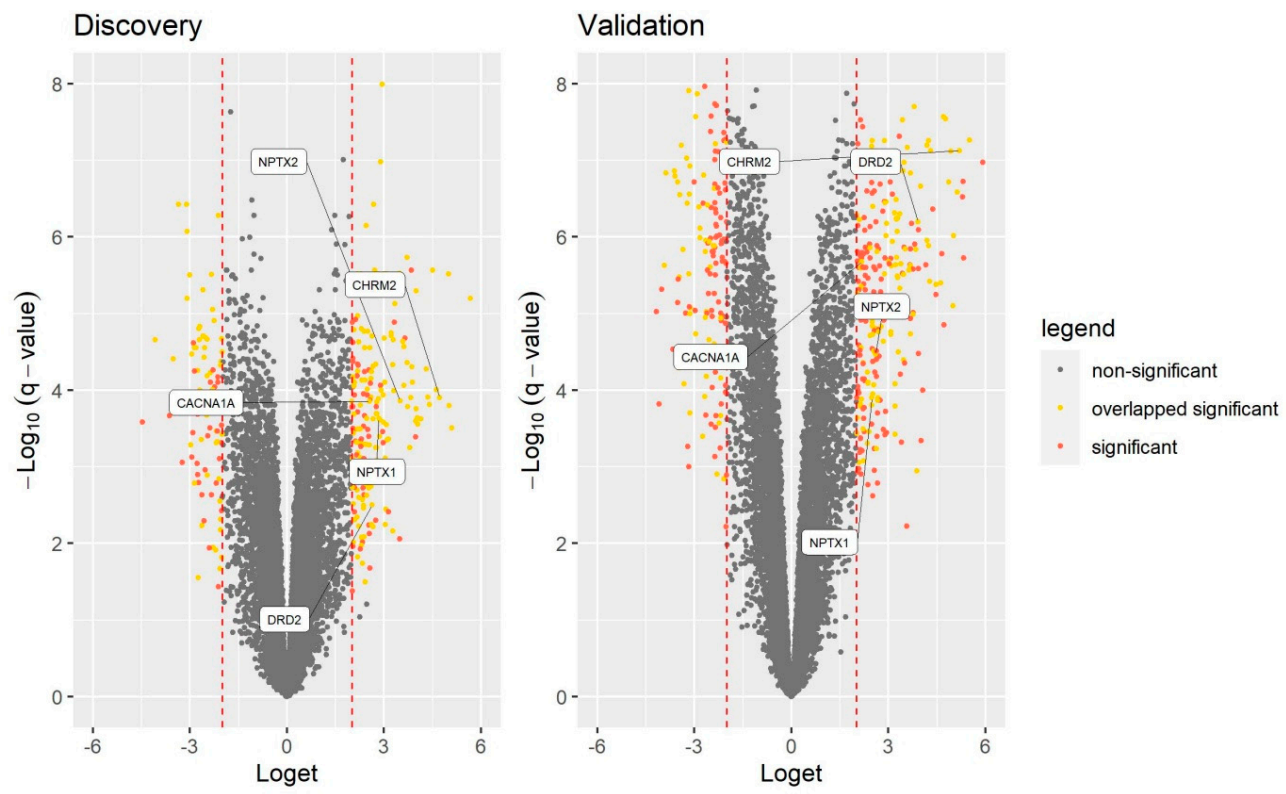

Figure 2. Volcano plots with overlapped and selected genes.

Table 4. RNA-seq and RT-qPCR results of selected genes.

\begin{tabular}{ccccccc}
\hline & \multicolumn{2}{c}{ Discovery } & \multicolumn{2}{c}{ Validation } & \multicolumn{2}{c}{ RT-qPCR } \\
\hline GENE & LOGET & $\boldsymbol{q}$ VALUE & LOGET & $\boldsymbol{q}$ VALUE & FC & $\boldsymbol{p}$ VALUE \\
\hline NPTX1 & 2.84 & $2.3 \times 10^{-4}$ & 2.49 & $1.3 \times 10^{-4}$ & 2.55 & 0.013 \\
NPTX2 & 3.50 & $1.4 \times 10^{-4}$ & 2.60 & $3.4 \times 10^{-5}$ & 7.34 & 0.015 \\
DRD2 & 2.63 & $3.2 \times 10^{-3}$ & 3.91 & $6.4 \times 10^{-7}$ & 6.08 & 0.016 \\
CHRM2 & 4.72 & $1.3 \times 10^{-4}$ & 5.19 & $7.5 \times 10^{-8}$ & 5.77 & 0.018 \\
CACNA1A & 2.55 & $1.4 \times 10^{-4}$ & 2.18 & $2.1 \times 10^{-6}$ & 4.68 & 0.024 \\
\hline
\end{tabular}

Loget: $\log _{2} \mathrm{FC}$ fibroid tumors relative to normal myometrium; FC: fold-change fibroid tumors relative to normal myometrium.
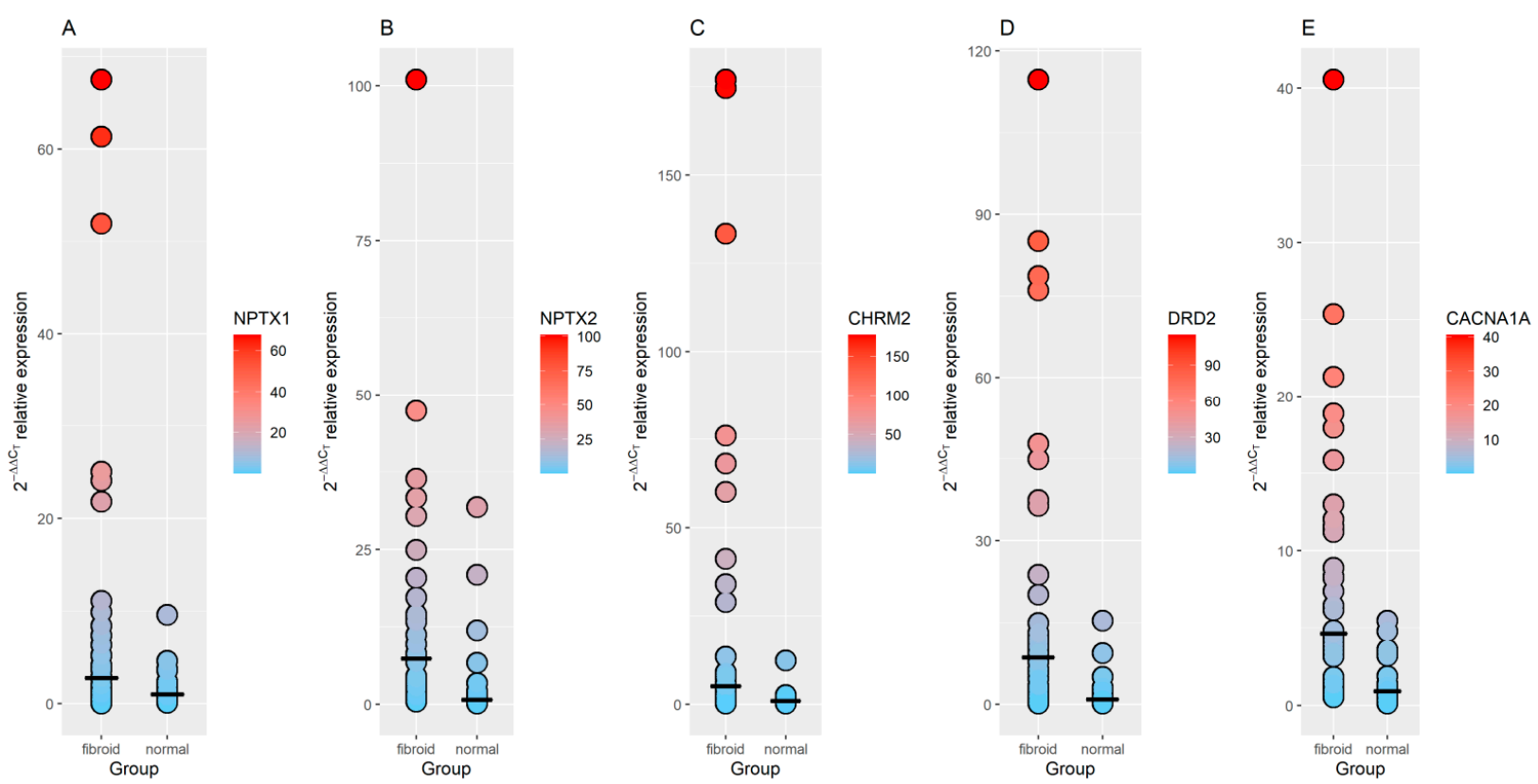

Figure 3. RT-qPCR results of selected genes. (A): NPTX1; (B): NPTX2; (C): CHRM2; (D): DRD2; (E): CACNA1A; fibroid: fibroid tumor; normal: normal myometrium. 


\subsection{Between-Gene Regulation of the Identified Genes and Prediction Value Estimation}

In order to assess the relationships between the identified genes, we performed correlation analyses. For that, discovery and validation RNA-seq datasets containing raw counts were merged, TPMs were calculated, and datasets were split into normal myometrium and fibroid tumor datasets. Using normal myometrium data, the correlation results showed an extensive interplay between all five genes (Figure $4 \mathrm{~A}$ ), whereas using fibroid tumor data, the correlation was retained only for NPTX1 and CACNA1A (Figure 4B). Moreover, in normal data, the correlation between NPTX1 and DRD2 was not observed, but in fibroid tumor data, the correlation between NPTX1 and DRD2 was observed. Subsequently, calculated TPMs and RT-qPCR results were used independently to assess the prediction value of selected genes using machine learning approach. For both RNA-seq and RT-qPCR obtained data, the Random Forest machine learning algorithm further confirmed the involvement of the five genes in uterine leiomyoma. For both datasets, the accuracy of prediction was 100\% and AUC: 1.

A

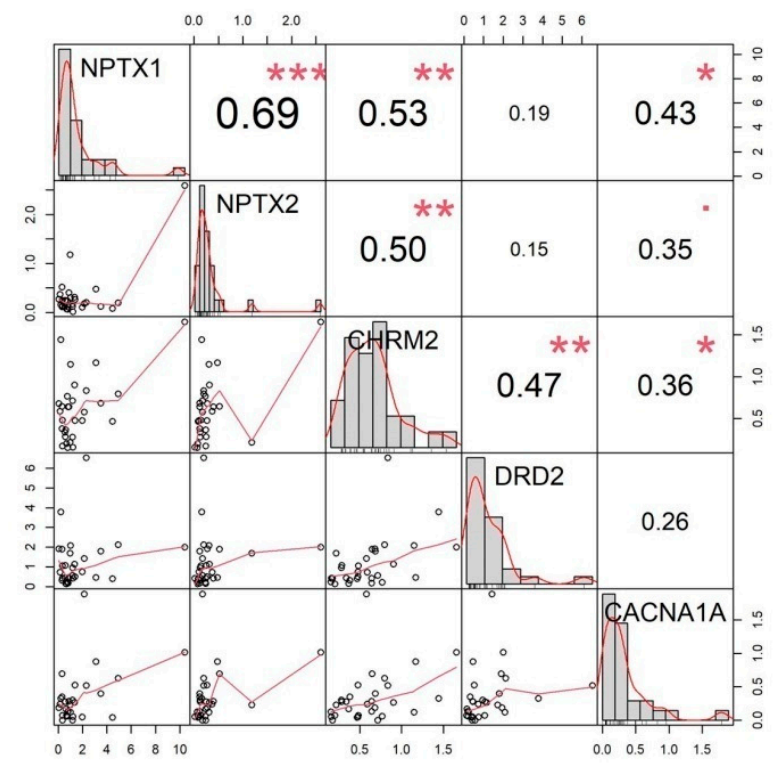

B

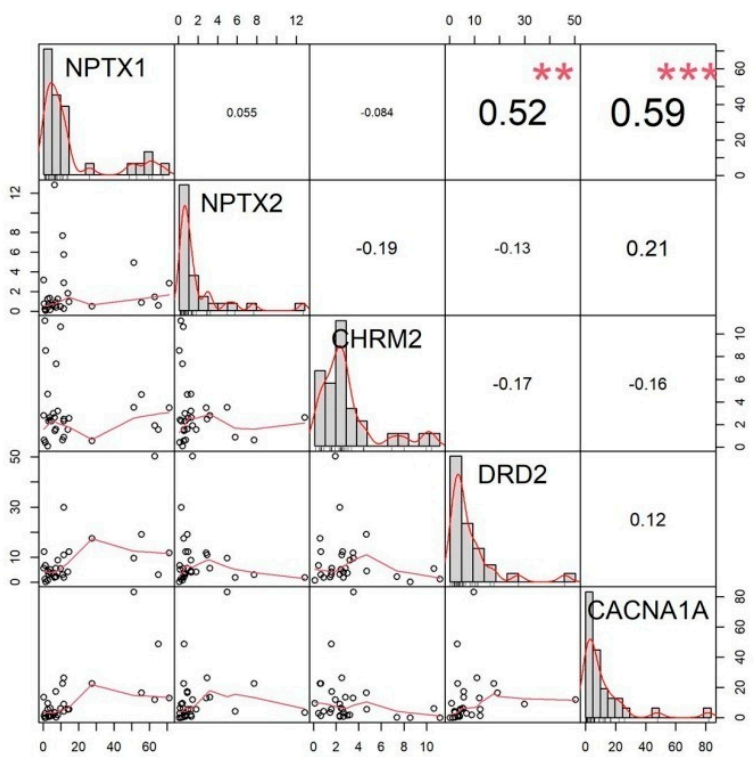

Figure 4. Correlation matrices. ${ }^{* *}$ : significant at $0.001{ }^{* *}$ : significant at 0.01 ; : significant at 0.05 . (A) normal myometrium; (B) fibroid tumor.

\subsection{Integration into Uterine Leiomyoma Meta-GWAs and Functional Analysis}

Subsequently, validated and confirmed expression results were integrated using previously published meta-analysis results achieved by Gallagher and colleagues [18]. We analyzed single nucleotide variants from meta-analysis summary statistics ranging $\pm 100 \mathrm{~kb}$ from previously identified differentially expressed genes, and we extracted the most significant signals at each region (Table 5). For genes NPTX1, NPTX2, CHRM2, DRD2 and CACNA1A, we found significant $3^{\prime}$ downstream variant rs9906819, $5^{\prime}$ upstream variant rs817758, $3^{\prime}$ downstream variant rs77571733, 5' upstream variant rs139711611 and intronic rs112605945 variant, respectively (Figure 5). No eQTL data were available for SNPs and selected genes. Using HaploReg, rs9906819 is listed as a genic enhancer by the Core 15-state model and 25-state model, and at H3K4me1_Enh and H3K27ac_Enh epigenetic chromatin state marks in various cells, there are also female skeletal muscle cells. Additionally, DNase hypersensitivity in fetal and psoas muscle is listed at rs9906819, and ChIP-seq evidence of NRSF binding exists at rs9906819. The PBX3 putative transcription factor binding motif is also listed as altered by the variant. SNP rs817758 is listed only at the H3K4me3_Pro epigenetic mark in lungs and spleen, and the YY1 putative transcription factor binding motif is altered by the variant. SNP rs77571733 is listed at H3K4me1_Enh in 
muscle, mesenchymal stem cells and lung fibroblasts, and POU2F2 putative transcription factor binding motif is listed as altered by the variant. SNP rs139711611 is listed at the H3K4me1_Enh epigenetic mark in mesoderm cultured cells and mammary epithelial cells. DNase hypersensitivity is also listed at rs139711611 in iPSCs. rs112605945 is listed as a genic enhancer by the Core 15-state model and 25-state model. DNase hyperactivity is also listed at the variant by 25-state model. The variant is also listed at H3K4me1_En, H3K4me3_Pro, H3K27ac_Enh and H3K9ac_Pro epigenetic chromatin state marks. CTCF, HNF4, NRSF, SP1 and Sin3Ak-20 putative transcription factor binding motifs are also listed as altered by the variant.

Table 5. Most significant SNPs in selected gene regions.

\begin{tabular}{ccccccccc}
\hline GENE & SNP & CONS & REF & ALT & EFFECT SIZE & ST ERROR & $p$ VALUE & N \\
\hline NPTX1 & rs9906819 & 3' downstream & A & C & 0.036 & 0.011 & 0.0013 \\
NPTX2 & rs817758 & 5' upstream & T & C & 0.085 & 0.029 & 0.0028 & 244324 \\
CHRM2 & rs77571733 & 3' downstream & A & G & -0.172 & 0.049 & 0.0004 & 238961 \\
DRD2 & rs139711611 & 5' upstream & A & C & -0.151 & 0.053 & 0.0044 & 238961 \\
CACNA1A & rs112605945 & intron variant & T & C & 0.078 & 0.026 & 0.0021 & 244324 \\
\hline
\end{tabular}

CONS: consequence; REF: reference allele; ALT: alternative allele; ST ERROR: standard error of meta-analysis; N: number of individuals at each observation.

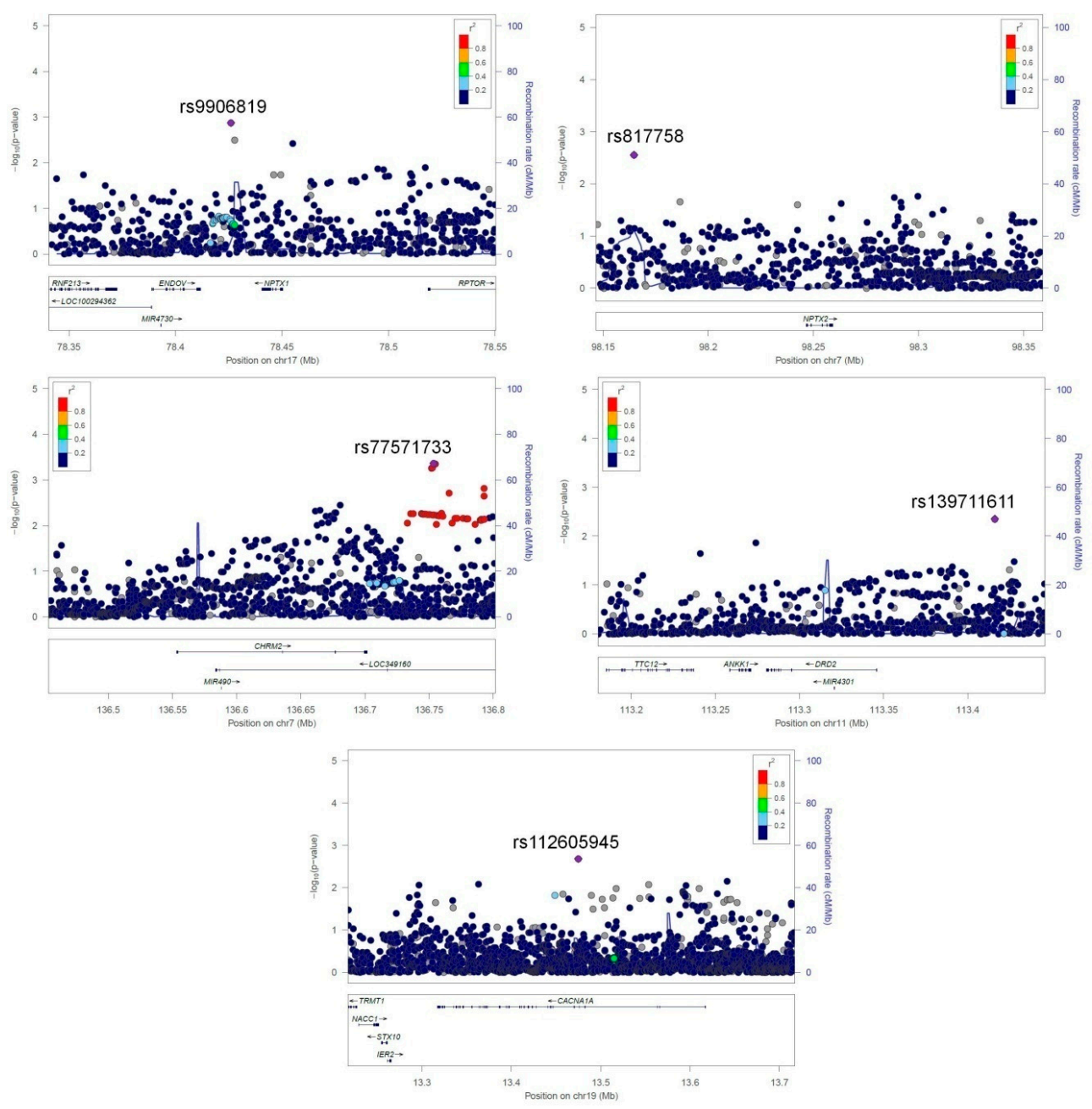

Figure 5. Regional Manhattan plots for selected gene regions from meta-analysis summary. 


\section{Discussion}

The biology of uterine leiomyoma is still not well understood, and recent state-ofthe-art integrative studies have shown that in addition to already established genetic stratification into four main subtypes, epigenetic malfunctions are recognized as important mechanisms of gene dysregulation in UL pathogenesis. Using the integration of transcriptomic and genetic data, the present study identified synaptic signaling genes NPTX1, NPTX2, CHRM2, DRD2 and CACNA1A, a unique subgroup of dysregulated genes in the biology of UL. To the best of our knowledge, this is the first time that synaptic signaling genes were observed to be associated with UL. GO analysis of the overlap of two independent RNA-seq analyses showed that NPTX1, NPTX2, CHRM2, DRD2 and $C A C N A 1 A$ were listed as significant for several enriched GO terms. These genes were subsequently validated using RT-qPCR in our own cohort consisting of 36 and 22 Slovenian patients and controls, respectively. The validation using Slovenian samples additionally confirmed the aforementioned genes. We observed that all five genes show significant upregulation in fibroid tumors relative to normal myometrium. However, Slovenian samples were not pair-matched, which presents a limitation of the study. Moreover, the present study also showed between-gene dysregulation when comparing the gene-gene interactions between normal myometrium and fibroid tumor. Correlation analysis has clearly shown that there is a shift in gene-gene regulations in fibroid tumors in terms of lost interplay. Moreover, based on correlation figures, it is clearly evident that the expression of the aforementioned genes is upregulated in fibroid tumors, ranging above 10 TPMs in comparison with normal myometrium, which ranges up to but not above 10 TPMs. The involvement of NPTX1, NPTX2, CHRM2, DRD2 and CACNA1A was additionally confirmed using a machine learning approach, which also confirmed the involvement of these genes in the biology of UL in two independent datasets obtained from RNA-seq analyses and an RT-qPCR experiment. As interesting as this finding may seem, there is evidence of association between the aforementioned genes and tumorogenesis in some cancers. NPTX1 (neuronal pentraxin 1) is a member of the pentraxin family and can bind various ligands, such as bacteria and also chromatin [37]. Molecular studies also reveal that NPTX1 functions by regulating both Nodal ligands and bone morphogenetic proteins (BMP) signaling via binding to TDGF1, which regulates pluripotency and neural differentiation [37]. Both Nodal ligands and BMP are also members of the TGF- $\beta$ family of ligands [38], which is in accordance with previous findings where TGF- $\beta$ was considered a key player gene in UL [39] and further extends previously identified pathways to synaptic signaling. Furthermore, NPTX1 was also identified as a novel epigenetic regulator that was associated with prognosis in lung cancer [40]. NPTX2 (neuronal pentraxin 2) is also a member of the highly conserved pentraxin protein group and was previously associated with neurodegenerative diseases [41]. Additionally, it was also previously observed that NPTX2 hypermethylation inhibits cell cycle arrest and apoptosis in gastric cancers via p53 suppression [42], which in turn suggests a possible association of NPTX2 with epigenetic regulation. Synaptic receptors CHRM2 (cholinergic receptor muscarinic 2) and DRD2 (dopamine receptor D2) have also been listed at significantly enriched terms in GO analysis. To the best of our knowledge, CHRM2 is not associated with conditions or functions that could put the gene on the radar in the development of UL pathogenesis. However, expression of CHRM2 is co-regulated with the expression of DRD2 in healthy myometrium but not in fibroid tumors, where correlation between these two genes has not been observed. In contrast to CHRM2, DRD2 has previously been associated with UL. It has been shown that $D R D 2$ codon $313\left({ }^{*}\right)$ T-related genotypes/alleles are associated with the presence of UL [43]. Interestingly, it was observed that the $C A C N A 1 A$ gene was listed at four enriched terms, where all four aforementioned genes were also present. $C A C N A 1 A$ gene encodes the voltage-dependent $\mathrm{P} / \mathrm{Q}$-type calcium channel subunit $\alpha-1 \mathrm{~A}$ and is widely expressed throughout the central nervous system. $C A C N A 1 A$ has previously been associated with a wide spectrum of neurological disorders [44]. Additionally, with epigenetic exploration, it was shown that methylation of $C A C N A 1 A$ is one of the markers for irradiation efficacy 
in oropharyngeal cancer [45]. Furthermore, $C A C N A 1 A$ has also been shown to have a high promoter methylation status in ovarian clear cell adenocarcinoma [46]. Methylation of $C A C N A 1 A$ is also associated with triple-negative breast cancer [47]. The aforementioned epigenetic associations of $C A C N A 1 A$ support the gene's involvement in epigenetics of tumorogenesis. Interestingly, a previous study demonstrated that innervation of the uterus is involved in multiple pathophysiological processes and suggests that autonomic innervations together with interstitial telocytes are involved in the microenvironmental imbalance of UL [48]. Moreover, the difference in adrenergic and cholinergic innervation between normal myometrium and fibroid tumors demonstrates the pivotal role of the neuronal component in the formation of UL [48]. These findings are further supported by the unique subset of genes identified by the present study. Subsequently, in order to further address and identify additional genetic-epigenetic landmarks involved in the formation of UL, we integrated a previously published genome-wide association meta-analysis summary [18] with identified gene regions ranging $\pm 100 \mathrm{~kb}$ from the genes. We selected the most significant SNPs in these regions and extracted four near gene variants (rs9906819, rs817758, rs77571733 and rs139711611) and one intron variant rs112605945 harboring in CACNA1A. None of the five variants was previously mentioned to be associated with any phenotype. Using HaploReg [34], we assessed the potential interplay between these variants and epigenetic landmarks. All five variants were listed at epigenetic chromatin state marks predicting enhancer and promoter histone modification. SNPs rs9906819 and rs139711611 were also listed as genic enhancers. Moreover, rs9906819, rs817758, rs77571733 and rs112605945 were flagged as altering a putative transcription factor binding motif. Additionally, rs9906819, rs139711611 and rs112605945 were listed at a DNase hypersensitivity chromatin structure. By establishing global chromatin states, histone modifications influence gene expression [49]. Histone residues can be acetylated or methylated [50], and acetylation is considered as a hallmark of active transcription [51]. Thus, the locations of the selected variants may help to elucidate the upregulation and dysregulation of the selected genes in UL tissue. In silico functional analysis using HaploReg clearly showed that the locations of the selected SNPs and corresponding effect alleles may influence the expression of the aforementioned genes. Moreover, these findings warrant a chromatin immunoprecipitation (ChIP) assay, as they are listed at specific chromatin states, protein binding sites and regulatory motifs. We hypothesize that the effect alleles of the selected variants may cause histone modifications in favor of upregulation of nearby genes and may additionally alter transcriptions binding motifs, promoting gene expression and thus the formation of the fibroid tumors. However, the role of the synaptic signaling genes in the biology of UL must be further elucidated. We believe that integration of independent studies and -omics may help to identify new signals, which are otherwise masked due to strong statistics signals obtained through homogenous sampling.

\section{Conclusions}

In summary, the present study identified a unique subgroup of dysregulated synaptic signaling genes in the biology of UL, adding to the complexity of the tumor biology. These findings may increase our understanding of the broad molecular interplay of signaling pathways and neuronal components in the formation of UL and further support epigenetic regulation as an important mechanism of uterine leiomyoma disease pathogenesis.

Supplementary Materials: The following are available online at https:/ / www.mdpi.com/article/10 .3390 /genes12081179/s1, Table S1: RNA-seq complete differential expression from discovery and validation cohort; Table S2: Raw Ct values from RT-qPCR.

Author Contributions: Conceptualization, J.K., M.G., I.B., M.P. and U.P.; methodology, J.K. and M.G.; formal analysis, J.K. and M.G.; investigation, J.K. and M.G.; data curation, J.K. and M.G.; writing-original draft preparation, J.K. and M.G.; writing-review and editing, J.K., M.G., I.B., M.P. and U.P.; visualization, J.K. and M.G.; supervision, U.P. All authors have read and agreed to the published version of the manuscript. 
Funding: The authors acknowledge the project was financially supported by the Slovenian Research Agency (research core funding No. P3-0067). The Slovenian Research Agency had no role in the design, execution, interpretation, or writing of the study.

Institutional Review Board Statement: The study protocol was approved by the National Medical Ethics Committee and the Institutional Review Board (KME 43/10/15).

Informed Consent Statement: Informed consent was obtained from all subjects involved in the study.

Data Availability Statement: Publicly available datasets were analyzed in this study. This data can be found here: (https:/ / www.ncbi.nlm.nih.gov/sra/SRP188330; https:/ / www.ncbi.nlm.nih.gov/ sra/SRP166862; https:/ / www.ncbi.nlm.nih.gov/sra/SRP217468; www.ebi.ac.uk/gwas/studies/ GCST009158 accessed on 5 May 2021). The data presented in this study are also available in Table S2.

Acknowledgments: The authors would like to thank the patients for their participation in this study. The authors would like to thank Natalia Hernandez-Pacheco, for advices and support.

Conflicts of Interest: Authors declare no conflict of interest. No benefits in any form have been received from any commercial party.

\section{References}

1. Stewart, E.A. Clinical practice. Uterine fibroids. N. Engl. J. Med. 2015, 372, 1646-1655. [CrossRef]

2. Stewart, E.A.; Laughlin-Tommaso, S.K.; Catherino, W.H.; Lalitkumar, S.; Gupta, D.; Vollenhoven, B. Uterine fibroids. Nat. Rev. Dis. Primers 2016, 2, 1-18. [CrossRef]

3. Bolt, H.M.; Janning, P.; Michna, H.; Degen, G.H. Comparative assessment of endocrine modulators with oestrogenic activity: I. Definition of a hygiene-based margin of safety (HBMOS) for xeno-oestrogens against the background of European developments. Arch. Toxicol. 2001, 74, 649-662. [CrossRef]

4. Malik, M.; Norian, J.; McCarthy-Keith, D.; Britten, J.; Catherino, W.H. Why Leiomyomas Are Called Fibroids: The Central Role of Extracellular Matrix in Symptomatic Women. Semin. Reprod. Med. 2010, 28, 169-179. [CrossRef]

5. Islam, S.; Ciavattini, A.; Petraglia, F.; Castellucci, M.; Ciarmela, P. Extracellular matrix in uterine leiomyoma pathogenesis: A potential target for future therapeutics. Hum. Reprod. Update 2018, 24, 59-85. [CrossRef]

6. Cramer, S.F.; Patel, A. The frequency of uterine leiomyomas. Am. J. Clin. Pathol. 1990, 94, 435-438. [CrossRef] [PubMed]

7. Marino, J.; Eskenazi, B.; Warner, M.; Samuels, S.; Vercellini, P.P.; Gavoni, N.; Olive, D. Uterine leiomyoma and menstrual cycle characteristics in a population-based cohort study. Hum. Reprod. 2004, 19, 2350-2355. [CrossRef] [PubMed]

8. Merrill, R.M. Hysterectomy surveillance in the United States, 1997 through 2005. Med. Sci. Monit. 2008, $14,24-31$.

9. Mehine, M.; Kaasinen, E.; Mäkinen, N.; Katainen, R.; Kämpjärvi, K.; Pitkänen, E.; Heinonen, H.-R.; Bützow, R.; Kilpivaara, O.; Kuosmanen, A.; et al. Characterization of Uterine Leiomyomas by Whole-Genome Sequencing. N. Engl. J. Med. 2013, 369, 43-53. [CrossRef] [PubMed]

10. Mäkinen, N.; Mehine, M.; Tolvanen, J.; Kaasinen, E.; Li, Y.; Lehtonen, H.J.; Gentile, M.; Yan, J.; Enge, M.; Taipale, M.; et al. MED12, the Mediator Complex Subunit 12 Gene, Is Mutated at High Frequency in Uterine Leiomyomas. Science 2011, 334, 252-255. [CrossRef]

11. Mehine, M.; Kaasinen, E.; Heinonen, H.-R.; Mäkinen, N.; Kämpjärvi, K.; Sarvilinna, N.; Aavikko, M.; Vähärautio, A.; Pasanen, A.; Bützow, R.; et al. Integrated data analysis reveals uterine leiomyoma subtypes with distinct driver pathways and biomarkers. Proc. Natl. Acad. Sci. USA 2016, 113, 1315-1320. [CrossRef] [PubMed]

12. Ciarmela, P.; Islam, S.; Reis, F.M.; Gray, P.C.; Bloise, E.; Petraglia, F.; Vale, W.; Castellucci, M. Growth factors and myometrium: Biological effects in uterine fibroid and possible clinical implications. Hum. Reprod. Update 2011, 17, 772-790. [CrossRef]

13. Lee, B.S.; Nowak, R.A. Human leiomyoma smooth muscle cells show increased expression of transforming growth factor- $\beta 3$ (TGF $\beta 3$ ) and altered responses to the antiproliferative effects of TGF $\beta$. J. Clin. Endocrinol. Metab. 2001, 86, 913-920. [CrossRef] [PubMed]

14. Dou, Q.; Zhao, Y.; Tarnuzzer, R.W.; Rong, H.; Williams, R.S.; Schultz, G.S.; Chegini, N. Suppression of transforming growth factor- $\beta$ (TGF $\beta$ ) and TGF $\beta$ receptor messenger ribonucleic acid and protein expression in leiomyomata in women receiving gonadotropin-releasing hormone agonist therapy. J. Clin. Endocrinol. Metab. 1996, 81, 3222-3230. [PubMed]

15. Sozen, I.; Arici, A. Interactions of cytokines, growth factors, and the extracellular matrix in the cellular biology of uterine leiomyomata. Fertil. Steril. 2002, 78, 1-12. [CrossRef]

16. Moyo, M.B.; Parker, J.B.; Chakravarti, D. Altered chromatin landscape and enhancer engagement underlie transcriptional dysregulation in MED12 mutant uterine leiomyomas. Nat. Commun. 2020, 11, 1-16. [CrossRef] [PubMed]

17. George, J.; Fan, H.; Johnson, B.; Carpenter, T.J.; Foy, K.K.; Chatterjee, A.; Patterson, A.L.; Koeman, J.; Adams, M.; Madaj, Z.B.; et al. Integrated Epigenome, Exome, and Transcriptome Analyses Reveal Molecular Subtypes and Homeotic Transformation in Uterine Fibroids. Cell Rep. 2019, 29, 4069-4085.e6. [CrossRef] 
18. Gallagher, C.S.; Mäkinen, N.; Harris, H.R.; Rahmioglu, N.; Uimari, O.; Cook, J.P.; Shigesi, N.; Ferreira, T.; Velez-Edwards, D.R.; Edwards, T.L.; et al. Genome-wide association and epidemiological analyses reveal common genetic origins between uterine leiomyomata and endometriosis. Nat. Commun. 2019, 10, 1-11. [CrossRef]

19. Ponomarenko, I.; Reshetnikov, E.; Polonikov, A.; Verzilina, I.; Sorokina, I.; Yermachenko, A.; Dvornyk, V.; Churnosov, M. Candidate Genes for Age at Menarche Are Associated with Uterine Leiomyoma. Front. Genet. 2021, 11, 512940. [CrossRef]

20. Hernandez-Pacheco, N.; Gorenjak, M.; Jurgec, S.; Corrales, A.; Jorgensen, A.; Karimi, L.; Vijverberg, S.J.; Berce, V.; Schieck, M.; Acosta-Herrera, M.; et al. Combined analysis of transcriptomic and genetic data for the identification of loci involved in glucocorticosteroid response in asthma. Allergy 2021, 76, 1238-1243. [CrossRef]

21. Gorenjak, M.; Zupin, M.; Jezernik, G.; Skok, P.; Potočnik, U. Omics data integration identifies ELOVL7 and MMD gene regions as novel loci for adalimumab response in patients with Crohn's disease. Sci. Rep. 2021, 11, 1-12. [CrossRef]

22. Liao, Y.; Smyth, G.; Shi, W. The Subread aligner: Fast, accurate and scalable read mapping by seed-and-vote. Nucleic Acids Res. 2013, 41, e108. [CrossRef]

23. Liao, Y.; Smyth, G.K.; Shi, W. featureCounts: An efficient general purpose program for assigning sequence reads to genomic features. Bioinformatics 2014, 30, 923-930. [CrossRef]

24. Robinson, M.D.; McCarthy, D.J.; Smyth, G.K. edgeR: A Bioconductor package for differential expression analysis of digital gene expression data. Bioinformatics 2010, 26, 139-140. [CrossRef]

25. Robinson, M.D.; Oshlack, A. A scaling normalization method for differential expression analysis of RNA-seq data. Genome Biol. 2010, 11, R25. [CrossRef] [PubMed]

26. Law, C.W.; Chen, Y.; Shi, W.; Smyth, G.K. voom: Precision weights unlock linear model analysis tools for RNA-seq read counts. Genome Biol. 2014, 15, R29. [CrossRef]

27. Ritchie, M.E.; Phipson, B.; Wu, D.; Hu, Y.; Law, C.W.; Shi, W.; Smyth, G.K. limma powers differential expression analyses for RNA-sequencing and microarray studies. Nucleic Acids Res. 2015, 43, e47. [CrossRef]

28. Shannon, P.; Markiel, A.; Ozier, O.; Baliga, N.S.; Wang, J.T.; Ramage, D.; Amin, N.; Schwikowski, B.; Ideker, T. Cytoscape: A Software Environment for Integrated Models of Biomolecular Interaction Networks. Genome Res. 2003, 13, 2498-2504. [CrossRef]

29. Bindea, G.; Mlecnik, B.; Hackl, H.; Charoentong, P.; Tosolini, M.; Kirilovsky, A.; Fridman, W.-H.; Pagès, F.; Trajanoski, Z.; Galon, J ClueGO: A Cytoscape plug-in to decipher functionally grouped gene ontology and pathway annotation networks. Bioinformatics 2009, 25, 1091-1093. [CrossRef]

30. Gorenjak, M.; Repnik, K.; Jezernik, G.; Jurgec, S.; Skok, P.; Potočnik, U. Genetic prediction profile for adalimumab response in Slovenian Crohn's disease patients. Z. Gastroenterol. 2019, 57, 1218-1225. [CrossRef]

31. Livak, K.J.; Schmittgen, T.D. Analysis of relative gene expression data using real-time quantitative PCR and the 2(-Delta Delta C(T)) Method. Methods 2001, 25, 402-408. [CrossRef]

32. Liaw, A.; Wiener, M. Classification and Regression by randomForest. $R$ News 2002, 2, 18-22.

33. Robin, X.; Turck, N.; Hainard, A.; Tiberti, N.; Lisacek, F.; Sanchez, J.-C.; Muller, M.J. pROC: An open-source package for R and S+ to analyze and compare ROC curves. BMC Bioinform. 2011, 12, 77. [CrossRef]

34. Ward, L.D.; Kellis, M. HaploReg: A resource for exploring chromatin states, conservation, and regulatory motif alterations within sets of genetically linked variants. Nucleic Acids Res. 2012, 40, D930-D934. [CrossRef]

35. Consortium, G. Erratum: Genetic effects on gene expression across human tissues. Nature 2018, 553, 530. [CrossRef] [PubMed]

36. Pruim, R.J.; Welch, R.P.; Sanna, S.; Teslovich, T.M.; Chines, P.S.; Gliedt, T.P.; Boehnke, M.; Abecasis, G.; Willer, C.J. LocusZoom: Regional visualization of genome-wide association scan results. Bioinformatics 2010, 26, 2336-2337. [CrossRef]

37. Boles, N.C.; Hirsch, S.E.; Le, S.; Corneo, B.; Najm, F.; Minotti, A.P.; Wang, Q.; Lotz, S.; Tesar, P.; Fasano, C.A. NPTX1 Regulates Neural Lineage Specification from Human Pluripotent Stem Cells. Cell Rep. 2014, 6, 724-736. [CrossRef] [PubMed]

38. Yeo, C.-Y.; Whitman, M. Nodal Signals to Smads through Cripto-Dependent and Cripto-Independent Mechanisms. Mol. Cell 2001, 7, 949-957. [CrossRef]

39. Paul, E.; Burns, G.; Carpenter, T.; Grey, J.; Fazleabas, A.; Teixeira, J. Transcriptome Analyses of Myometrium from Fibroid Patients Reveals Phenotypic Differences Compared to Non-Diseased Myometrium. Int. J. Mol. Sci. 2021, 22, 3618. [CrossRef]

40. Zhou, C.; Qin, Y.; Xie, Z.; Zhang, J.; Yang, M.; Li, S.; Chen, R. NPTX1 is a novel epigenetic regulation gene and associated with prognosis in lung cancer. Biochem. Biophys. Res. Commun. 2015, 458, 381-386. [CrossRef]

41. Libiger, O.; Shaw, L.M.; Watson, M.H.; Nairn, A.C.; Umaña, K.L.; Biarnes, M.C.; Canet-Avilés, R.M.; Jack, C.R., Jr.; Breton, Y.A.; Cortes, L. Longitudinal CSF proteomics identifies NPTX2 as a prognostic biomarker of Alzheimer's disease. Alzheimers Dement. 2021. [CrossRef]

42. Xu, G.; Fan, L.; Zhao, S.; OuYang, C. Neuronal pentraxin II (NPTX2) hypermethylation promotes cell proliferation but inhibits cell cycle arrest and apoptosis in gastric cancer cells by suppressing the p53 signaling pathway. Bioengineered 2021, 12, 1311-1323. [CrossRef]

43. Hsieh, Y.-Y.; Chang, C.-C.; Bau, D.-T.; Tsai, F.-J.; Tsai, C.-H.; Chen, C.-P. The p21 codon 31*C- and DRD2 codon $313 * T-r e l a t e d$ genotypes/alleles, but not XRCC1 codon 399, hOGG1 codon 326, and DRD1-48 polymorphisms, are correlated with the presence of leiomyoma. Fertil. Steril. 2009, 91, 869-877. [CrossRef]

44. Zhang, L.; Wen, Y.; Zhang, Q.; Chen, Y.; Wang, J.; Shi, K.; Du, L.; Bao, X. CACNA1A Gene Variants in Eight Chinese Patients With a Wide Range of Phenotypes. Front. Pediatr. 2020, 8, 577544. [CrossRef] 
45. Kurokawa, T.; Nakagawa, T.; Matsusaka, K.; Fukuyo, M.; Mima, M.; Misawa, K.; Rahmutulla, B.; Ikeda, J.-I.; Hanazawa, T.; Okamoto, Y.; et al. Establishment of epigenetic markers to predict irradiation efficacy against oropharyngeal cancer. Cancer Sci. 2020, 111, 1407-1416. [CrossRef]

46. Ho, C.-M.; Huang, C.-J.; Huang, C.-Y.; Wu, Y.-Y.; Chang, S.-F.; Cheng, W.-F. Promoter Methylation status of HIN-1 associated with outcomes of ovarian clear cell adenocarcinoma. Mol. Cancer 2012, 11, 53. [CrossRef]

47. Branham, M.T.; Marzese, D.; Laurito, S.R.; Gago, F.E.; Orozco, J.I.; Tello, O.M.; Vargas-Roig, L.M.; Roqué, M. Methylation profile of triple-negative breast carcinomas. Oncogenesis 2012, 1, e17. [CrossRef] [PubMed]

48. Aleksandrovych, V.; Kurnik-Łucka, M.; Bereza, T.; Białas, M.; Pasternak, A.; Cretoiu, D.; Walocha, J.A.; Gil, K. The Autonomic Innervation and Uterine Telocyte Interplay in Leiomyoma Formation. Cell Transplant. 2019, 28, 619-629. [CrossRef]

49. Barski, A.; Cuddapah, S.; Cui, K.; Roh, T.-Y.; Schones, D.E.; Wang, Z.; Wei, G.; Chepelev, I.; Zhao, K. High-Resolution Profiling of Histone Methylations in the Human Genome. Cell 2007, 129, 823-837. [CrossRef]

50. Park, J.-A.; Kim, A.-J.; Kang, Y.; Jung, Y.-J.; Kim, H.K.; Kim, K.-C. Deacetylation and methylation at histone H3 lysine 9 (H3K9) coordinate chromosome condensation during cell cycle progression. Mol. Cells 2011, 31, 343-349. [CrossRef] [PubMed]

51. Karmodiya, K.; Krebs, A.R.; Oulad-Abdelghani, M.; Kimura, H.; Tora, L. H3K9 and H3K14 acetylation co-occur at many gene regulatory elements, while $\mathrm{H} 3 \mathrm{~K} 14 \mathrm{ac}$ marks a subset of inactive inducible promoters in mouse embryonic stem cells. BMC Genom. 2012, 13, 424. [CrossRef] 